\title{
Improved High Order Methods Using Boundary Layer Detection for a Singular Perturbation Problem
}

\author{
Weiqun Zhang \\ Department of Mathematics, Wright State University, Lake Campus, Ohio, USA.
}

How to cite this paper: Weiqun Zhang. (2020) Improved High Order Methods Using Boundary Layer Detection for a Singular Perturbation Problem. Journal of Applied Mathematics and Computation, 4(4), 189-194.

DOI: $10.26855 /$ jamc.2020.12.010

Received: October 20, 2020

Accepted: November 25, 2020

Published: December 11, 2020

${ }^{*}$ Corresponding author: Weiqun Zhang, Department of Mathematics, Wright State University, Lake Campus, Ohio, USA.

Email: weiqun.zhang@wright.edu

\begin{abstract}
A singular perturbation problem is solved with improved high order methods using boundary layer detection theorems. The stability and convergence, independent of the singular perturbation parameter, is numerically verified.
\end{abstract}

\section{Keywords}

Singular Perturbation, Differential Equations, Boundary Layers

We consider the singular perturbation problem,

$$
\begin{aligned}
& \varepsilon u^{\prime \prime}-u u^{\prime}=0 \quad x \in(-1,1), \\
& u(-1)=0 \quad \text { and } \quad u(1)=-1 .
\end{aligned}
$$

from Chang [1], O’Malley [2] and Miller [3]. Using the boundary layer detection theorem in Zhang [4], an exponential boundary layer of width proportional the singular perturbation parameter can be found to the left boundary. Because of the presence of the boundary layer, the numerical methods from Keller [5] will have to be adjusted accordingly. Choudhury [6] and Ilicasu [7] solved the problem using nonstandard high order methods on a uniform mesh. With the theory of weak formulation in Lax [8], Zhang [9] proposed test functions fitting the exponential boundary layer to solve the singular perturbation. In this paper, the singular perturbation problem is solved with improved high order methods based on the boundary layer detection theorem. Furthermore, a sixth order method is developed and verified numerically.

\section{An improvement on the $4^{\text {th }}$ order method}

Let $\omega=\frac{1}{\varepsilon}$ where $\varepsilon$ is the singular perturbation parameter in the problem (1). Then we rewrite the second order derivative and compute the third and fourth order derivatives,

$$
\begin{aligned}
& u^{\prime \prime}=\omega u u^{\prime}, \\
& u^{\prime \prime \prime}=\omega\left(u^{\prime}\right)^{2}+\omega u u^{\prime \prime}, \text { and }
\end{aligned}
$$


$u^{(4)}=2 \omega u^{\prime} u^{\prime \prime}+\omega u^{\prime} u^{\prime \prime}+\omega u u^{\prime \prime}=3 \omega u^{\prime} u^{\prime \prime}+\omega^{2} u\left(u^{\prime}\right)^{2}+\omega^{2} u^{2} u^{\prime \prime}=\omega^{2} u\left(u^{\prime}\right)^{2}+\left(3 \omega u^{\prime}+\omega^{2} u^{2}\right) u^{\prime \prime}$

Setting $A_{3}=\omega u_{i}^{\prime}, B_{3}=\omega u_{i}$, and $A_{4}=\omega^{2} u_{i} u_{i}^{\prime}, B_{4}=3 \omega u_{i}^{\prime}+\omega^{2} u_{i}^{2}$, we get $u_{i}^{(3)}=A_{3} u_{i}^{\prime}+B_{3} u_{i}^{\prime \prime}, u_{i}^{(4)}=A_{4} u_{i}^{\prime}+B_{4} u_{i}^{\prime \prime}$.

For the purpose of simplicity, we continue to use the following new notations,

$$
A^{* *}=\frac{h^{4} A_{4}}{24}, B^{* *}=h+\frac{h^{3} A_{3}}{6}, C^{* *}=\frac{h^{2}}{2}+\frac{h^{4} B_{4}}{24}, D^{* *}=\frac{h^{3} B_{3}}{6}
$$

To improve the accuracy of the process, we develop the fourth order finite differences to approximate the singular perturbation problem (1).

$\varepsilon u_{i}^{\prime \prime}-u_{i} u_{i}^{\prime} \approx c_{3}^{* *} u_{i+1}+c_{2}^{* *} u_{i}+c_{1}^{* *} u_{i-1}$ where

$$
\begin{gathered}
c_{3}^{* *}=\frac{-u_{i} D^{* *}-\varepsilon B^{* *}+\varepsilon A^{* *}+u_{i} C}{2\left(A^{* *} D^{* *}-B^{* * *} C^{* *}\right)}, \\
c_{1}^{* *}=\frac{-u_{i} D^{* *}-\varepsilon B^{* *}-\left(\varepsilon A^{* *}+u_{i} C^{* *}\right)}{2\left(A^{* *} D^{* *}-B^{* *} C^{* *}\right)}, \\
c_{2}^{* *}=-\left(c_{3}^{* *}+c_{1}^{* *}\right)=\frac{u_{i} D^{* *}+\varepsilon B^{* *}}{A^{* *} D^{* *}-B^{* *} C^{* *}} .
\end{gathered}
$$

In Ilicasu [7], the derivative $u_{i}^{\prime}$ in $A_{3}, A_{4}$ and $B_{4}$ is replaced with $u_{i}^{\prime}=\frac{u_{i+1}-u_{i-1}}{2 h}$. We now use the fourth order finite differences to establish a higher order approximation to the derivative $u_{i}^{\prime}$. For $i=1,2, \ldots, N-1$, let the first derivative be, $u_{i}^{\prime}=c_{3} u_{i+1}+c_{2} u_{i}+c_{1} u_{i-1}$ where $c_{3}, c_{2}$ and $c_{1}$ are constants.

By Taylor series expansion, we have

$$
\begin{aligned}
u_{i}^{\prime} & =c_{3}\left(u_{i}+h u_{i}^{\prime}+\frac{h^{2}}{2} u_{i}^{\prime \prime}+\frac{h^{3}}{2} u_{i}^{\prime \prime \prime}+\frac{h^{7}}{24} u_{i}^{(4)}+\ldots\right) \\
& +c_{2} u_{i}+c_{1}\left(u_{i}-h_{i}^{\prime}+t \frac{h^{2}}{2} u_{i}^{\prime \prime}-\frac{h^{3}}{6} u_{i}^{\prime \prime \prime}+\frac{h^{4}}{24} u_{i}^{(4)}+\ldots\right) \\
& \approx c_{3}\left\{u_{i}+h u^{\prime}+\frac{h^{2}}{2} u_{i}^{\prime \prime}+\frac{h^{3}}{6}\left(\omega u_{i}^{\prime 2}+\omega u_{i} u_{i}^{\prime \prime}\right)+\frac{h^{4}}{24}\left[\omega^{2} u_{i} u_{i}^{\prime 2}+\left(3 \omega u_{i}^{\prime}+\omega^{2} u_{i}^{2}\right) u_{i}^{\prime \prime}\right]\right\} \\
& +c_{2} u_{i}+c_{1}\left\{u_{i}-h_{i}^{\prime}+t \frac{h^{2}}{2} u_{i}^{\prime \prime}-\frac{h^{3}}{6}\left(\omega u_{i}^{\prime 2}+\omega u_{i} u_{i}^{\prime \prime}\right)+\frac{h^{4}}{24}\left[\omega^{2} u_{i} u_{i}^{\prime 2}+\left(3 \omega u_{i}^{\prime}+\omega^{2} u_{i}^{2}\right) u_{i}^{\prime \prime}\right]\right\} \\
& =\left(c_{3}+c_{2}+c_{1}\right) u_{i}+\left[\left(c_{3}-c_{1}\right)\left(h+\frac{h^{3}}{6} \omega u_{i}^{\prime}\right)+\left(c_{3}+c_{1}\right) \frac{h^{4}}{24} \omega^{2} u_{i} u_{i}^{\prime}\right] u_{i}^{\prime} \\
& +\left[\left(c_{3}+c_{1}\right)\left(\frac{h^{2}}{2}+\frac{h^{4}}{24} 3 \omega u_{i}^{\prime}+\frac{h^{4}}{24} \omega^{2} u_{i}^{2}\right)+\left(c_{3}-c_{1}\right) \frac{h^{3}}{6} \omega u_{i}\right] u_{i}^{\prime \prime} .
\end{aligned}
$$

Matching the corresponding coefficients, we obtain the following system of equations

$$
\begin{aligned}
& c_{3}+c_{2}+c_{1}+0, \\
& \left(c_{3}-c_{1}\right)\left(h+\frac{h^{3}}{6} \omega u_{i}^{\prime}\right)+\left(c_{3}+c_{1}\right) \frac{h^{4}}{24} \omega^{2} u_{i} u_{i}^{\prime}=1, \\
& \left(c_{3}+c_{1}\right)\left(\frac{h^{2}}{2}+\frac{h^{4}}{24} 3 \omega u_{i}^{\prime}+\frac{h^{4}}{24} \omega^{2} u_{i}^{\prime 2}\right)+\left(c_{3}-c_{1}\right) \frac{h^{3}}{6} \omega u_{i}=0 .
\end{aligned}
$$

To understand the system better, we create more notations, 


$$
\begin{aligned}
& A=\frac{h^{4}}{24} \omega^{2} u_{i} u_{i}^{\prime}, \\
& B=h+\frac{h^{3}}{6} \omega u_{i}^{\prime}, \\
& C=\frac{h^{2}}{2}+\frac{h^{4}}{24} 3 \omega u_{i}^{\prime}+\frac{h^{4}}{24} \omega^{2} u_{i}^{\prime 2}, \\
& D=\frac{h^{3}}{6} \omega u_{i},
\end{aligned}
$$

It is clear the system of equations is equivalent to

$$
\begin{aligned}
& c_{3}+c_{2}+c_{1}=0, \\
& \left(c_{3}+c_{1}\right) A+\left(c_{3}-c_{1}\right) B=1, \\
& \left(c_{3}+c_{1}\right) C+\left(c_{3}-c_{1}\right) D=0,
\end{aligned}
$$

of which, $c_{3}+c_{1}$ and $c_{3}-c_{1}$ are,

$$
\begin{aligned}
& c_{3}+c_{1}=\frac{D}{A D-B C}, \\
& c_{3}-c_{1}=\frac{-C}{A D-B C} .
\end{aligned}
$$

Therefore, we get

$$
\begin{aligned}
& c_{3}=\frac{D-C}{2(A D-B C)}, \\
& c_{1}=\frac{D+C}{2(A D-B C)}, \\
& c_{2}=-\left(c_{3}+c_{1}\right)=\frac{-D}{A D-B C} .
\end{aligned}
$$

The error term is $\frac{h}{120}\left(c_{3} u^{(5)}\left(\eta_{1}\right)+c_{1} u^{(5)}\left(\eta_{2}\right)\right)$ where $\eta_{1}, \eta_{2} \in\left[x_{i}-h, x_{i}+h\right]$.

Now $c_{3}$ and $c_{1}$ are updated to the fourth order accuracy. The improvement of the method is verified by numerical experiments.

\section{The $6^{\text {th }}$ Order Method}

The second improvement is to add more terms from the Taylor series. We expand the $u_{i+1}$ and $u_{i-1}$ up to the sixth order derivatives. The following is a development of the sixth order method. The fifth order method is developed by dropping the sixth order derivative terms. We consider the fifth order and sixth order derivatives from the singular perturbation problem (1):

and

$$
\begin{aligned}
u^{(5)} & =3 \omega u^{\prime \prime 2}+3 \omega u^{\prime} u^{\prime \prime \prime}+\omega^{2} u^{\prime} u^{\prime 2}+2 \omega^{2} u u^{\prime \prime} u^{\prime \prime}+2 \omega^{2} u u^{\prime \prime} u^{\prime \prime}+\omega^{2} u^{2} u^{\prime \prime \prime} \\
& =3 \omega u^{\prime \prime 2}+3 \omega u^{\prime}\left(\omega u^{\prime 2}+\omega u u^{\prime \prime}\right)+\omega^{2} u^{\prime 3}+4 \omega^{2} u u^{\prime} u^{\prime \prime}+\omega^{2} u^{2}\left(\omega u^{\prime 2}+\omega u u^{\prime \prime}\right) \\
& =3 \omega u^{\prime \prime 2}+3 \omega^{2} u^{\prime 3}+3 \omega^{2} u u^{\prime} u^{\prime \prime}+\omega^{2} u^{\prime 3}+4 \omega^{2} u u^{\prime} u^{\prime \prime}+\omega^{2} u^{2} u^{\prime 2}+\omega^{3} u^{3} u^{\prime \prime} \\
& =3 \omega u^{\prime \prime 2}+4 \omega^{2} u^{\prime 3}+7 \omega^{2} u u^{\prime} u^{\prime \prime}+\omega^{3} u^{2} u^{\prime 2}+\omega^{3} u^{3} u^{\prime \prime} \\
& =\left(4 \omega^{2} u^{\prime 2}+\omega^{3} u^{2} u^{\prime}\right) u^{\prime}+\left(3 \omega u^{\prime \prime}+7 \omega^{2} u u^{\prime}+\omega^{3} u^{3}\right) u^{\prime \prime}
\end{aligned}
$$




$$
\begin{aligned}
u^{(6)}= & 6 \omega u^{\prime \prime} u^{\prime \prime \prime}+12 \omega^{2} u^{\prime 2} u^{\prime \prime}+7 \omega^{2}\left[\left(u^{\prime 2}+u u^{\prime \prime}\right) u^{\prime \prime}+u u^{\prime} u^{\prime \prime}\right] \\
& +2 \omega^{3} u u^{3}+2 \omega^{3} u^{2} u^{\prime} u^{\prime \prime}+3 \omega^{3} u^{2} u^{\prime \prime} u^{\prime \prime}+\omega^{3} u^{3} u^{\prime \prime \prime} \\
& =6 \omega u^{\prime \prime}\left(\omega u^{\prime 2}+\omega u u^{\prime \prime}\right)+12 \omega^{2} u^{\prime 2} u^{\prime \prime}+7 \omega^{2}\left[u^{\prime 2} u^{\prime \prime}+u u^{\prime \prime 2}+u u^{\prime}\left(\omega u^{\prime 2}+\omega u u^{\prime \prime}\right)\right] \\
& +2 \omega^{3} u u^{\prime 3}+5 \omega^{3} u^{2} u^{\prime} u^{\prime \prime}+\omega^{3} u^{3}\left(\omega u^{\prime 2}+\omega u u^{\prime \prime}\right) \\
& =6 \omega^{2} u^{\prime 2} u^{\prime \prime}+6 \omega^{2} u u^{\prime 2}+12 \omega^{2} u^{\prime 2} u^{\prime \prime}+7 \omega^{2} u^{\prime 2} u^{\prime \prime}+7 \omega^{2} u u^{\prime \prime}+7 \omega^{3} u u^{\prime 3}+7 \omega^{3} u^{2} u^{\prime} u^{\prime \prime} \\
& +2 \omega^{3} u u^{3}+5 \omega^{3} u^{2} u^{\prime} u^{\prime \prime}+\omega^{4} u^{3} u^{\prime 2}+\omega^{4} u^{4} u^{\prime \prime} \\
& =25 \omega^{2} u^{\prime 2} u^{\prime \prime}+13 \omega^{2} u u^{\prime 2}+7 \omega^{3} u u^{\prime 3}+2 \omega^{3} u u^{\prime 3}+12 \omega^{3} u^{2} u^{\prime \prime} u^{\prime \prime}+\omega^{4} u^{3} u^{\prime 2}+\omega^{4} u^{4} u^{\prime \prime} \\
& =\left(9 \omega^{3} u u^{\prime 2}+\omega^{4} u^{3} u^{\prime}\right) u^{\prime}+\left(25 \omega^{2} u^{\prime 2}+13 \omega^{2} u u^{\prime \prime}+12 \omega^{3} u^{2} u^{\prime}+\omega^{4} u^{4}\right) u^{\prime \prime}
\end{aligned}
$$

For simplicity, we rewrite the derivatives

$$
\begin{aligned}
& u_{i}^{(3)}=A_{3} u_{i}^{\prime}+B_{3} u_{i}^{\prime \prime} \text { where } A_{3}=\omega u_{i}^{\prime}, B_{3}=\omega u_{i}, \\
& u_{i}^{(4)}=A_{4} u_{i}^{\prime}+B_{4} u_{i}^{\prime \prime} \text { where } A_{4}=\omega^{2} u_{i} u_{i}^{\prime}, B_{4}=3 \omega u_{i}^{\prime}+\omega^{2} u_{i}^{2}, \\
& u_{i}^{(5)}=A_{5} u_{i}^{\prime}+B_{5} u_{i}^{\prime \prime} \text { where } A_{5}=4 \omega^{2} u_{i}^{\prime 2}+\omega^{3} u_{i}^{2} u_{i}^{\prime}, B_{5}=3 \omega u_{i}^{\prime \prime}+7 \omega^{2} u_{i} u_{i}^{\prime}+\omega^{3} u_{i}^{3},
\end{aligned}
$$

and

$$
\begin{aligned}
& u_{i}^{(6)}=A_{6} u_{i}^{\prime}+B_{6} u_{i}^{\prime \prime} \text { where } A_{6}=9 \omega^{3} u_{i} u_{i}^{\prime 2}+\omega^{4} u_{i}^{3} u_{i}^{\prime} \text {, and } \\
& B_{6}=25 \omega^{3} u_{i}^{\prime 2}+13 \omega^{2} u_{i} u_{i}^{\prime \prime}+12 \omega^{3} u_{i}^{2} u_{i}^{\prime}+\omega^{4} u_{i}^{4}
\end{aligned}
$$

We write

$$
\varepsilon u_{i}^{\prime \prime}-u_{i} u_{i}^{\prime}=c_{3}^{*} u_{i+1}+c_{2}^{*} u_{i}+c_{1}^{*} u_{i-1} \text {, }
$$

where $c_{3}^{*}, c_{2}^{*}$ and $c_{1}^{*}$ are constants. By Taylor series expansion, we obtain

$$
\begin{aligned}
\varepsilon u_{i}^{\prime \prime}-u_{i} u_{i}^{\prime}= & c_{3}^{*} u_{i+1}+c_{2}^{*} u_{i}+c_{1}^{*} u_{i-1} \\
\approx & c_{3}^{*}\left[u_{i}+h u_{i}^{\prime}+\frac{h^{2}}{2} u_{i}^{\prime \prime}+\frac{h^{3}}{6} u_{i}^{\prime \prime \prime}+\frac{h^{4}}{24} u_{i}^{(4)}+\frac{h^{2}}{120} u_{i}^{(5)}+\frac{h^{6}}{720} u_{i}^{(6)}\right] \\
& +c_{2}^{*} u_{i}+c_{1}^{*}\left[u_{i}-h_{i}^{\prime}+\frac{h^{2}}{2} u_{i}^{\prime \prime}-\frac{h^{3}}{6} u_{i}^{\prime \prime \prime}+\frac{h^{4}}{24} u_{i}^{(4)}-\frac{h^{5}}{120} u_{i}^{(5)}+\frac{h^{6}}{720} u_{i}^{(6)}\right] \\
= & c_{3}^{*}\left[u_{i}+h u_{i}^{\prime}+\frac{h^{2}}{2} u_{i}^{\prime \prime}+\frac{h^{3}}{6}\left(A_{3} u_{i}^{\prime}+B_{3} u_{i}^{\prime \prime}\right)+\frac{h^{2}}{24}\left(A_{4} u_{i}^{\prime}+B_{4} u_{i}^{\prime \prime}\right)+\frac{h^{5}}{120}\left(A_{5} u_{i}^{\prime}+B_{5} u_{i}^{\prime \prime}\right)+\frac{h^{6}}{720}\left(A_{6} u_{i}^{\prime}+B_{6} u_{i}^{\prime \prime}\right)\right] \\
& +c_{2}^{*} u_{i}+c_{1}^{*}\left[u_{i}-h_{i}^{\prime}+t \frac{h^{2}}{2} u_{i}^{\prime \prime}-\frac{h^{3}}{6}\left(A_{3} u_{i}^{\prime}+B_{3} u_{i}^{\prime \prime}\right)+\frac{h^{2}}{24}\left(A_{4} u_{i}^{\prime}+B_{4} u_{i}^{\prime \prime}\right)-\frac{h^{5}}{120}\left(A_{5} u_{i}^{\prime}+B_{5} u_{i}^{\prime \prime}\right)+\frac{h^{6}}{720}\left(A_{6} u_{i}^{\prime}+B_{6} u_{i}^{\prime \prime}\right)\right] \\
= & c_{3}^{*}\left[u_{i}+\left(h+\frac{h^{3} A_{3}}{6}+\frac{h^{4} A_{4}}{24}+\frac{h^{5} A_{5}}{120}+\frac{h^{6} A_{6}}{720}\right) u_{i}^{\prime}+\left(\frac{h^{2}}{2}+\frac{h^{3} B_{3}}{6}+\frac{h^{4} B_{4}}{24}+\frac{h^{5} B_{5}}{120}+\frac{h^{6} B_{6}}{720}\right) u_{i}^{\prime \prime}\right]+c_{2}^{*} u_{i} \\
& +c_{1}^{*}\left[u_{i}+\left(-h-\frac{h^{3} A_{3}}{6}+\frac{h^{4} A_{4}}{24}-\frac{h^{5} A_{5}}{120}+\frac{h^{6} A_{6}}{720}\right) u_{i}^{\prime}+\left(\frac{h^{2}}{2}-\frac{h^{3} B_{3}}{6}+\frac{h^{4} B_{4}}{24}-\frac{h^{5} B_{5}}{120}+\frac{h^{6} B_{6}}{720}\right) u_{i}^{\prime \prime}\right] \\
= & \left(c_{3}^{*}+c_{2}^{*}+c_{1}^{*}\right) u_{i}+\left[\left(c_{3}^{*}+c_{1}^{*}\right)\left(\frac{h^{4} A_{4}}{24}+\frac{h^{6} A_{6}}{720}\right)+\left(c_{3}^{*}-c_{1}^{*}\right)\left(h+\frac{h^{3} A_{3}}{6}+\frac{h^{5} A_{5}}{120}\right)\right] u_{i}^{\prime} \\
& +\left[\left(c_{3}^{*}+c_{1}^{*}\right)\left(\frac{h^{2}}{2}+\frac{h^{4} B_{4}}{24}+\frac{h^{6} B_{6}}{720}\right)+\left(c_{3}^{*}-c_{1}^{*}\right)\left(\frac{h^{3} B_{3}}{6}+\frac{h^{5} B_{5}}{120}\right)\right] u_{i}^{\prime \prime}
\end{aligned}
$$


Matching the corresponding coefficients of both sides, we get the following system of equation in terms of $c_{3}^{*}, c_{2}^{*}$ and $c_{1}^{*}:$

$$
\begin{aligned}
& c_{3}^{*}+c_{2}^{*}+c_{1}^{*}=0, \\
& \left(c_{3}^{*}+c_{1}^{*}\right) A^{*}+\left(c_{3}^{*}-c_{1}^{*}\right) B^{*}=-u_{i}, \\
& \left(c_{3}^{*}+c_{1}^{*}\right) C^{*}+\left(c_{3}^{*}-c_{1}^{*}\right) D^{*}=\varepsilon,
\end{aligned}
$$

where

$$
A^{*}=\frac{h^{4} A_{4}}{24}+\frac{h^{6} A_{6}}{720}, B^{*}=h+\frac{h^{3} A_{3}}{6}+\frac{h^{5} A_{5}}{120}, C^{*}=\frac{h^{2}}{2}+\frac{h^{4} B_{4}}{24}+\frac{h^{6} B_{6}}{720}, D^{*}=\frac{h^{3} B_{3}}{6}+\frac{h^{5} B_{5}}{120} .
$$

Note that the derivatives contained in $A_{3}, A_{4}, A_{5}$ and $B_{3}, B_{4}, B_{5}$ are replaced with the following:

$$
u_{i}^{\prime}=\frac{u_{i+1}-u_{i-1}}{2 h}, u_{i}^{\prime \prime}=\frac{u_{i+1}-2 u_{i}+u_{i-1}}{h^{2}} \text {. }
$$

Thus, we get

Therefore, the solution is

$$
\begin{aligned}
& c_{3}^{*}+c_{1}^{*}=\frac{-u_{i} D^{*}-\varepsilon B^{*}}{A^{*} D^{*}-B^{*} C^{*}}, \\
& c_{3}^{*}-c_{1}^{*}=\frac{\varepsilon A^{*}+u_{i} C^{*}}{A^{*} D^{*}-B^{*} C^{*}} .
\end{aligned}
$$

$$
\begin{gathered}
C_{3}^{*}=\frac{-u_{i} D^{*}-\varepsilon B^{*}+\varepsilon A^{*}+u_{i} C^{*}}{2\left(A^{*} D^{*}-B^{*} C^{*}\right)}, \\
C_{1}^{*}=\frac{-u_{i} D^{*}-\varepsilon B^{*}-\left(\varepsilon A^{*}+u_{i} C^{*}\right)}{2\left(A^{*} D^{*}-B^{*} C^{*}\right)}, \\
c_{2}^{*}=-\left(c_{3}^{*}+c_{1}^{*}\right)=\frac{u_{i} D^{*}+\varepsilon B^{*}}{A^{*} D^{*}-B^{*} C^{*}} .
\end{gathered}
$$

The error term is $\frac{h^{2}}{5040}\left(c_{3} u^{(7)}\left(\eta_{3}\right)+c_{1} u^{(7)}\left(n_{4}\right)\right)$ where $\eta_{3}, \eta_{4} \in\left[x_{i}-h, x_{i}+h\right]$.

The results are improved as shown in the tables. We compare the numerical results among different methods. For the methods of Choudhury [6] and Ilicasu [7], a uniform mesh is used with $\mathrm{N}=2,000$ mesh points. For the improved $4^{\text {th }}$ order, $5^{\text {th }}$ order and $6^{\text {th }}$ order methods, the number $N_{n}$ of mesh points on the non-boundary layer domain is 170 and the number $N_{b}$ of points on the boundary layer is 300 . In addition to the improved accuracy, the computing cost is reduced significantly thanks to fewer number of mesh points.

Table 1. Maximal error comparison among different methods $\varepsilon=0.01$

\begin{tabular}{cccc}
\hline Method & Number of Points & Number of Iterations & Max Error \\
\hline Choudhury's Method [6] & 2,000 & Not known & $2.91 * 10^{-2}$ \\
$2^{\text {nd }}$ order of Ilicasu [7] & 2,000 & 3,201 & $2.61 * 10^{-4}$ \\
$4^{\text {th }}$ order of Ilicasu [7] & 2,000 & 3,152 & $1.00 * 10^{-5}$ \\
Improved $4^{\text {th }}$ order method of this paper & 470 & 697 & $8.40 * 10^{-5}$ \\
$5^{\text {th }}$ order method of this paper & 470 & 697 & $1.34 * 10^{-7}$ \\
$6^{\text {th }}$ order method of this paper & 470 & 697 & $7.71 * 10^{-8}$ \\
\hline
\end{tabular}

For the methods of this paper, the tolerance of iteration is set at $10^{-10}$.

The improved high order methods work well for much smaller values of the singular perturbation parameter. The con- 
vergence of the improved fourth order method is shown in Table 2, with the smallest values of singular perturbation parameter $\varepsilon=10^{-12}$.

Table 2. The convergence of the improved fourth order method

\begin{tabular}{cccc}
\hline Number of Points & \multicolumn{2}{c}{ Maximal Error } \\
& $\varepsilon=10^{-5}$ & $\varepsilon=10^{-10}$ & $3.56^{*} 10^{-4}$ \\
$N=350\left(N_{n}=200, N_{b}=150\right)$ & $3.53 * 10^{-4}$ & $3.53 * 10^{-4}$ & $1.94 * 10^{-4}$ \\
$N=400\left(N_{n}=200, N_{b}=200\right)$ & $1.91 * 10^{-4}$ & $1.94 * 10^{-4}$ & $1.35 * 10^{-4}$ \\
$N=450\left(N_{n}=200, N_{b}=250\right)$ & $1.20^{*} 10^{-4}$ & $1.22 * 10^{-4}$ & $9.71 * 10^{-5}$ \\
$N=500\left(N_{n}=200, N_{b}=300\right)$ & $8.39 * 10^{-5}$ & $8.40^{-5} 10^{-5}$ & 9 \\
\hline
\end{tabular}

\section{References}

[1] K. W. Chang and F. A. Howes. (1984). Nonlinear Singular Perturbation Phenomena: Theory and Application. Spring-Verlag.

[2] R. E. O’Malley Jr. (1974). Introduction to Singular Perturbations. Academic Press, New York.

[3] J. J. H. Miller, E. O’Riordan, G. I. Shishkin. (1996). Fitted numerical methods for singular perturbation problems. World Scientific.

[4] W. Zhang, D. H. Schultz, and Lin. (2008). Numerical Solutions of Linear and Nonlinear Singular Perturbation Problems. Computers \& Math. Applic., Vol. 55, Issue 11, June 2008, pp. 2574-2592.

[5] H. B. Keller. (1968). Numerical Methods for Two-Point Boundary Value Problems. Blaisdell Publishing Company.

[6] S. R. Choudhury. (1996). Nonstandard Difference Schemes of Nonlinear Singular Perturbation Problems. Int. J. of Applied Sc. \& Computations, Vol. 2, pp. 375-392, February 1996.

[7] F. O. Ilicasu and D. H. Schultz. (2002). High Order Methods for Singular Perturbation Problems. Computers Math. Applic., Vol. 47, pp. 391-417.

[8] P. D. Lax and A. N. Milgram. (n.d.). Contributions to the Theory of Partial Differential Equations. Annals of Mathematics Studies, Princeton University Press.

[9] W. Zhang. (2019). A Uniformly Convergent Numerical Method Using Weak Formulation for Singularly Perturbed Differential Equations. Journal of Mathematics and System Science, Vol. 9, No. 1, pp. 1-6, doi: $10.17265 / 2159-5291$. 